\title{
Discussion on the choosing of Lyapunov function for adaptive parameter identification problem
}

\author{
Yuqiang $\mathrm{Jin}^{1}$, Junwei Lei ${ }^{2}$ and Huali $\mathrm{Wu}^{2}$ \\ ${ }^{1}$ Department of Training, Naval aeronautical and astronautical University \\ Yanti, 264001 \\ ${ }^{2}$ Department of Control engineering, Naval aeronautical and astronautical University \\ Yanti, 264001 \\ ajinyuqiang1024@126.com
}

Keywords: adaptive control, parameter identification, one order system, final value, stability Abstract: The selection of Lyapunov function is depth studied with adaptive parameter identification, where a kind of simple first order system with two unknown parameters is taken as an example. The study shows that the selection of Lyapunov function is completely free, and the proportion of the parameter identification and the steady-state error part can be matched arbitrarily. But the above two coefficients should be set to be positive, and the different proportion coefficient setting method will lead to different result of steady state error and parameter identification.

\section{Introduction}

Parameter identification ${ }^{[1-4]}$ provides a kind of solution for the system parameters calculation. In 1962, Zadeh first time proposed the word system identification. According to the definition of Zadeh: "the system identification is based on the input and output data, from a set of given model classes, to determine a system equivalent to the test model.

According to the identification theory, the identification method can be divided into two categories $^{[5-8]}$, the classical identification method and the modern identification method. Adaptive parameter identification ${ }^{[9-12]}$ is a small branch of modern identification method.

In this paper, we discuss the free degree problem of Lyapunov function selection with the adaptive parameter identification method, and the coefficient of the parameter identification and error convergence of the Lyapunov function can be arbitrarily selected. The final study shows that, as long as both parameters are positive, the Lyapunov function is completely free. But with different proportion, the parameter identification and error convergence effect are different.

\section{Problem description}

An one order system can be written as:

$$
\dot{x}=a_{1} x+a_{2} \sin x+u
$$

where $a_{1}$ and $a_{2}$ are unknown constant parameter, the goal is designing a controller $u=h\left(x, \hat{a}_{1}, \hat{a}_{2}\right)$ such that 
the system state $x$ can trace the expected value $x^{d}$ and $\hat{a}_{i}$ can converge to $a_{i}$.

\section{Design of adaptive identification controller}

An ordinary adaptive control method is used as follows, define a error variable as $z_{1}=x_{1}-x_{1}^{d}$, then

$$
\dot{z}_{1}=\dot{x}_{1}-\dot{x}_{1}^{d}=a_{1} x+a_{2} \sin x+u
$$

Design state feedback control law as:

$$
u=-\hat{a}_{1} x-\hat{a}_{2} \sin x-\sum_{i=1}^{n} k_{i} f_{i}\left(z_{1}\right)
$$

Choose $n=5, \quad k_{i}>0$

$$
\begin{gathered}
f_{1}\left(z_{1}\right)=z_{1}, \quad f_{2}\left(z_{1}\right)=z_{1}^{3}, \quad f_{3}\left(z_{1}\right)=z_{1}^{1 / 3} \\
f_{4}\left(z_{1}\right)=\frac{z_{1}}{\left|z_{1}\right|+\varepsilon}, \quad \varepsilon=0.2 \\
f_{5}\left(z_{1}\right)=\frac{1-e^{-\tau z_{1}}}{1+e^{-\tau z_{1}}}, \quad \tau=0.5
\end{gathered}
$$

where $f_{3}\left(z_{1}\right)$ is Terminal attractor, and $f_{5}\left(z_{1}\right)$ is Sigmoid function, $f_{4}\left(z_{1}\right)$ and $f_{5}\left(z_{1}\right)$ are both bounded, Obviously, $f_{i}\left(z_{1}\right)$ satisfies $z_{1} f_{i}\left(z_{1}\right) \geq 0$, then

$$
\dot{z}_{1}=\tilde{a}_{1} x+\tilde{a}_{2} \sin x-\sum_{i=1}^{n} k_{i} f_{i}\left(z_{1}\right)
$$

where the error variable $\tilde{a}_{i}$ can be defined as:

$$
\tilde{a}_{1}=a_{1}-\hat{a}_{1}, \quad \tilde{a}_{2}=a_{2}-\hat{a}_{2}
$$

Design regulating law as:

$$
\dot{\hat{a}}_{1}=\Gamma_{1} z_{1} x, \quad \dot{\hat{a}}_{2}=\Gamma_{2} z_{1} \sin x
$$

where $\hat{a}_{i}$ is unknown estimated parameter value, choose initial value $\hat{a}_{i}(0)=0$, then

$$
\dot{\tilde{a}}_{1}=-\dot{\hat{a}}_{1}, \quad \dot{\tilde{a}}_{2}=-\dot{\hat{a}}_{2}
$$

Choose a Lyapunov function as:

$$
V=\frac{1}{2} z_{1}^{2}+\frac{1}{2 \Gamma_{1}} \tilde{a}_{1}^{2}+\frac{1}{2 \Gamma_{2}} \tilde{a}_{2}^{2}
$$

Then 


$$
\dot{V}=z_{1} \dot{z}_{1}+\frac{1}{\Gamma_{1}} \tilde{a}_{1} \dot{\tilde{a}}_{1}+\frac{1}{\Gamma_{2}} \tilde{a}_{2} \dot{\tilde{a}}_{2}
$$

Then:

$$
\begin{aligned}
& \dot{V}=z_{1} \tilde{a}_{1} x+z_{1} \tilde{a}_{2} \sin x-\sum_{i=1}^{n} k_{i} z_{1} f_{i}\left(z_{1}\right)-\frac{1}{\Gamma_{1}} \tilde{a}_{1} \Gamma_{1} z_{1} x-\frac{1}{\Gamma_{2}} \tilde{a}_{2} \Gamma_{2} z_{1} \sin x \\
& =-\sum_{i=1}^{n} k_{i} z_{1} f_{i}\left(z_{1}\right) \leq 0
\end{aligned}
$$

So according to Lyapunov theory we get $z_{1} \rightarrow 0$.

\section{Parameter identification result analysis}

When $z_{1} \rightarrow 0$, where $u=-\hat{a} x$, then

$$
\dot{z}_{1}=a_{1} x-\hat{a}_{1} x+a_{2} \sin x-\hat{a}_{2} \sin x=\tilde{a}_{1} x+\tilde{a}_{2} \sin x
$$

When $z_{1} \rightarrow 0$, there is $\dot{z}_{1} \rightarrow 0$, then there is $\dot{z}_{1}=\tilde{a}_{1} x+\tilde{a}_{2} \sin x=0$, so the parameter can not be identified.

\section{Numerical simulation}

According to above model, choose unknown parameters as $a_{1}=5, a_{2}=-3$, set expected value as $x_{1}^{d}=2$, and write $m$ language program as follows:

clc; clear; $\mathrm{k} 11=-10 ; \mathrm{k} 12=-5 ; \mathrm{k} 13=-5 ; \mathrm{k} 14=-5 ; \mathrm{k} 15=-5 ; \mathrm{k} 16=-5 ;$ esten $1=0.2 ;$ taox $=3$;

And use Simulink to construct a program structure as following figures:

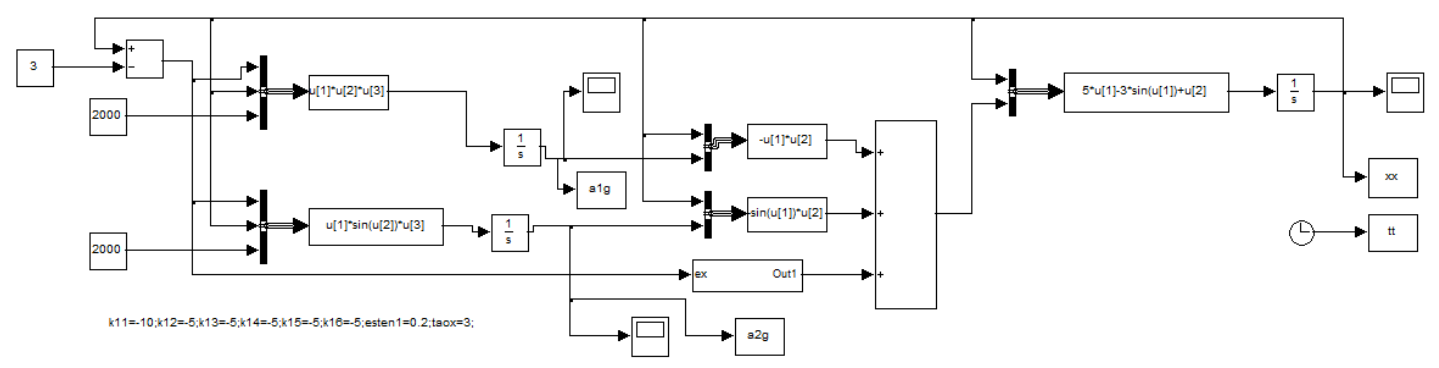

Fig.1 Figure of Simulink Program

Write program as follows to plot figure:

figure(1); plot $\left(t t, x x, k^{\prime}\right) ; x l a b e l\left(' t / s^{\prime}\right) ; y l a b e l\left(' s t a t e x x^{\prime}\right)$;

figure(2); plot( $\left.t t, a 1 g, k^{\prime}\right) ; x l a b e l\left(' t / s^{\prime}\right) ; y l a b e l(' s t a t e ~ a 1 g ')$;

figure(3);plot(tt,a2g, 'k');xlabel('t/s');ylabel('state a2g');

Choose the speed of unknown parameter as $\Gamma_{1}=-50, \Gamma_{2}=50$, then the simulation result is as following figures: 


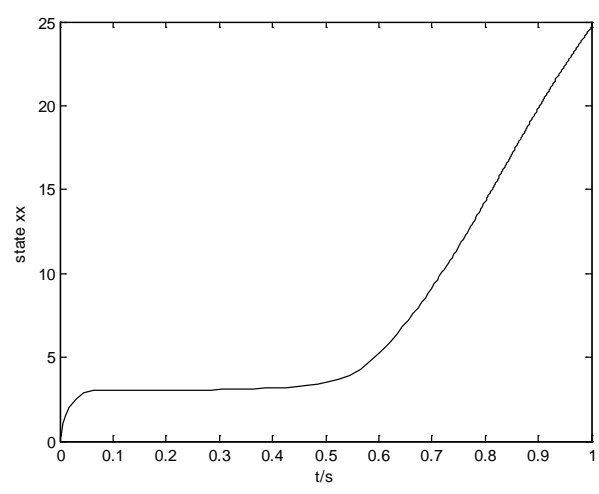

Fig.2 state x1

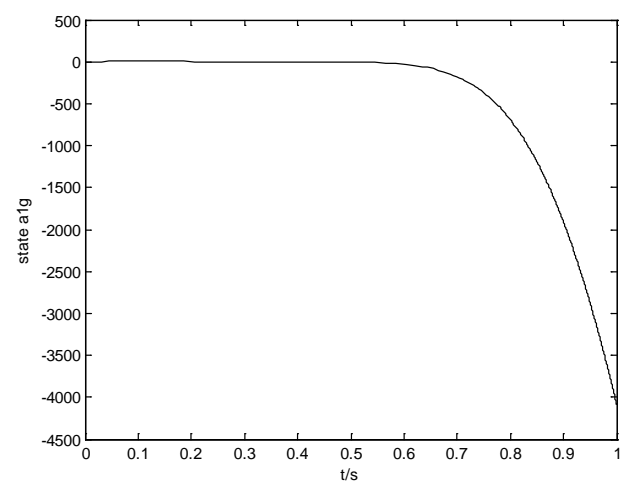

Fig.3 state a1g

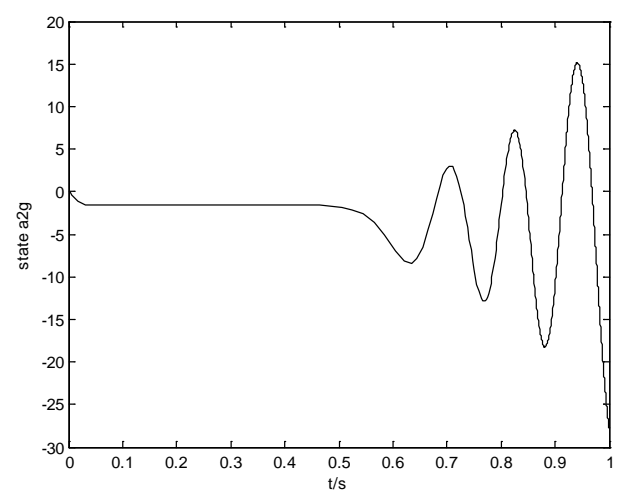

Fig.4 state a2g

The simulation results show that the identification speed of improved integral control is lower than no-integral control.

\section{Conclusion}

According to above discussion, a conclusion can be made as follows: the choosing of Lyapunov function is totally free. And in other word, the speed of adaptive law is free but it is need to be positive. But if the speed of adaptive law is too big, then the system response will has oscillations. And if the speed is too slow, then the steady state error of the system will converged to zero to quick such that the parameters are not identified yet. In all, a slow speed is good for the identification of unknown parameters and the performance of control is not depended on the adaptive parameter identification very much.

\section{Reference}

[1] Maurizio C, Marcello P, Giansalvo, et al. A new experimental application of least-squares techniques for the estimation of the induction motor parameters[J]. IEEE Transactions on Industry Applications, 2003, 39(5):1247-1256.

[2] Raj M B, Alexander G P, Hamid A T, Neural speed filtering for sensorless induction motor drives[J]. Control Engineering Practice, 2004, 12(6): 687-706.

[3] Huang K S, Kent W, Wu Q, et al. Parameter identification of an induction machine using genetic algorithms[C] // Proceedings of the 1999 IEEE International Symposium on Computer Aided Control System Design. [S. L.]: [s. N.],1999: 510-515.

[4]E. Mooij, Direct model reference adaptive control of a winged reentry vehicle [A], In: AIAA 9th International Space Planes and Hypersonic Systems and Technologies Conference [C], Norfolk: AIAA, 99-45484 
[5]Mooij E.Numerical investigation of model reference adaptive control for hypersonic aircraft [J]. Journal of Guidance, Control, and Dynamics, 2001, 24(2):315-323.

[6]E. Mooij, Model reference adaptive guidance for reentry trajectory tracking[A], In: AIAA Guidance, Navigation, and Control Conference and Exhibit [C], Providence: AIAA, 2004-4775

[7]Mooij E and Barkana I, Stability Analysis of an Adaptive Guidance and Control System applied to a Winged Re-entry Vehicle, Proceedings of the 2005 AIAA Guidance, Navigation, and ControI Conference and Exhibit,San Francisco, CA, AIAA 2005:6290

[8]S. Juliana, Q. P. Chu, J. A. Mulder, T. J. van Baten, Flight control of atmospheric re-entry vehicle with non-linear dynamic inversion [A], In: AIAA Guidance, Navigation, and Control Conference and Exhibit [C], Providence: AIAA, 2004-5330

[9]Johnson MD, Calise AJ, and Johnson EN, Evaluation of an Adaptive Method for Launch Vehicle Flight Control [A], In: AIAA Guidance, Navigation and Control Conference[C], Austin: AIAA, 2003: 1-11.

[10]Johnson MD, Calise AJ, and Johnson EN, Further Evaluation of an Adaptive Method for Launch Vehicle Flight Control [A], In: AIAA Guidance, Navigation and Control Conference [C], Providence: AIAA, 2004: 1-17.

[11]Baohua Lian, Hyochoong Bang, J. E. Hurtado, Adaptive Backstepping control based autopilot design for reentry vehicle [A], In: AIAA Guidance, Navigation, and Control Conference and Exhibit [C], Providence: AIAA, 2004-5328

[12]J. Fatemi, E. Mooij, S. P. Gurav, Reentry vehicle design optimization with intergrated trajectory uncertainties [A], In: AIAA 13th International Space Planes and Hypersonic Systems and Technologies Conference [C], AIAA, 2005-3386 